\title{
Characterization of charge trapping processes in fully-depleted UNIBOND SOI MOSFET subjected to $\gamma$-irradiation
}

\author{
Y. Houk, ${ }^{1}$ A.N. Nazarov, ${ }^{1}$ V.I. Turchanikov, ${ }^{1}$ V.S. Lysenko, ${ }^{1}$ \\ S. Andriaensen, ${ }^{2}$ and D. Flandre ${ }^{2}$ \\ ${ }^{1} V$. Lashkaryov Institute of Semiconductor Physics, NAS of Ukraine, \\ 45, prospect Nauky, 03028 Kyiv, Ukraine, \\ ${ }^{2}$ DICE, Universite Catholique de Louvain, Louvain-la-Neuve, Belgium
}

\begin{abstract}
An investigation of radiation effect on edgeless accumulation mode (AM) $p$-channel and fully-depleted enhancement mode (EM) $n$-channel MOSFETs, fabricated on UNIBOND silicon on insulatior wafers (SOI), is presented in the paper. Characterization of trapped charge in the gate and buried oxides of the devices was performed by measuring only the front-gate transistors. It was revealed that the irradiation effect on EM $n$-MOSFET is stronger than that on AM p-MOSFET. Radiationinduced positive charge in the buried oxide proved to invert back interface what causes back channel creation in EM $n$-MOSFET but no such effect in AM $p$-MOSFET has been not observed. The effect of improving the quality of both interfaces for small irradiation doses is demonstrated.
\end{abstract}

Keywords: SOI, MOSFET, radiation, small-dose improvement.

Manuscript received 06.10.05; accepted for publication 29.03.06.

\section{Introduction}

Thin-film SOI EM and AM MOSFETs are very attractive as basic elements of CMOS integrated circuits for high-temperature applications [1]. But due to the charge coupling effect [2] and the presence of thick buried oxide (BOX) in SOI wafers these elements have to be less radiation-resistant to a total dose. The purpose of the present work is to study the radiation effect on SOI MOSFETs that are devoted to high-temperature applications - such investigation has not yet been performed. Besides, we use the double derivation method [3-5] applied only to front-gate characteristics of SOI MOSFETs to extract the charges generated both in the gate and buried oxides during irradiation, in contrast to usual extraction of radiation charge generated in the BOX, which needs carrying out back-gate SOI MOSFETs measurements [6, 7].

\section{Experimental}

Fully-depleted (FD) EM $n$ - and AM $p$-MOSFETs (Fig. 1a) fabricated in the same chip using UNIBOND SOI material have been investigated. After device processing the BOX, silicon film and gate oxide thicknesses were 360,80 and $38 \mathrm{~nm}$, respectively. The doping channel concentration was $5 \cdot 10^{16} \mathrm{~cm}^{-3}$. Edgeless devices with $L / W=3 / 172 \mu \mathrm{m}$ were considered in order to avoid edge effects. For irradiation, the ${ }^{60} \mathrm{Co}$ gammaray source with the flux of $390 \mathrm{rad} / \mathrm{s}$ has been used. The MOSFETs were shorted during irradiation.

Transfer characteristics (drain current vs gate voltage $I_{D} V_{G_{1}}$ ) as a function of the bias applied to the silicon substrate $V_{G_{2}}$ (see Fig. 1b) were measured at room temperature in the linear regime $\left(V_{D}=0.1 \mathrm{~V}\right)$. First and second derivatives of $I_{D} V_{G_{1}}$ characteristics were calculated numerically.

\section{Theory}

The most clearly the effect of the radiation on the SOI MOSFETs is revealed in the change of transfer characteristics of the devices: as seen from Fig. 2, the transfer characteristics are shifted to the left, both for FD EM (Fig. 2a) and AM MOSFETs (Fig. 2b). In other words, the threshold voltage of the characteristics decreases with increasing the radiation dose regardless of the type of the irradiated transistor. As will be shown below, careful investigation of transfer characteristics, measured only on the front-gate, allows to determine all possible parameters of radiation-induced charge trapping in the gate and BOX of the transistors. 

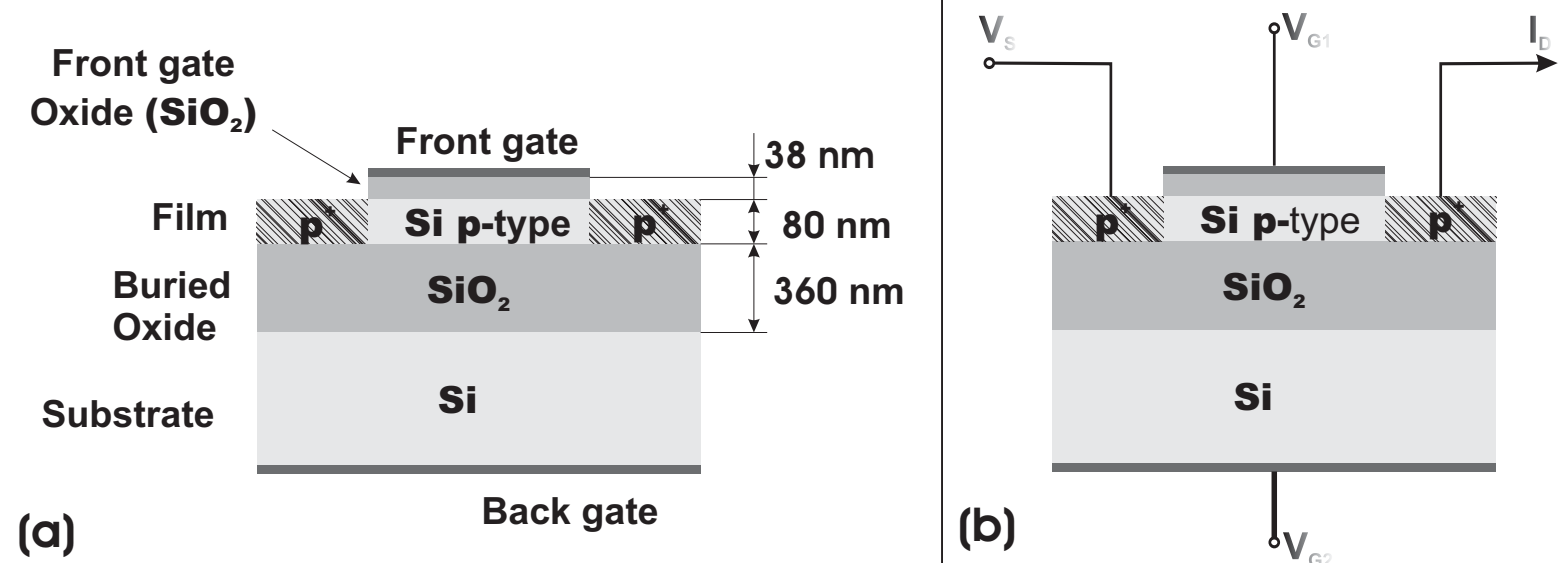

Fig. 1. SOI AM p-MOSFET cross-section (a); measurement scheme used (b).

In Figs $3 a$ and $3 a^{\prime}$, showed are the transfer characteristics $I_{D} V_{G_{1}}$ for high-dose irradiated FD EM $n$-MOSFETs and AM $p$-MOSFETs at room temperature. Correspondingly below them, the plots of their first derivatives, or transconductances, are depicted (Figs 3b and $\left.3 b^{\prime}\right)$. The front-gate transconductance curves allow to calculate the mobilities of the charge carriers both in the front and back interfaces of the transistor. The mobility of the charge carriers in the front channel, $\mu_{1}$, of the MOSFETs is calculated from the maximum transconductance under the depletion condition at the BOX/silicon film interface, $g_{\max }$ (see Fig. 3b) [1]:

$\mu_{1}=\frac{L C_{\mathrm{ox}_{1}} g_{\mathrm{max}}}{W V_{S}}$,

where $L$ and $W$ are, respectively, the length and the width of the channel, $C_{\mathrm{ox}_{1}}$ is the capacitance of the gate oxide, and $V_{S}$ is the source voltage. The mobility in the back channel $\mu_{2}$ is calculated from the plateau observed in the front-channel transconductance $g_{\text {plateau }}$ (see Fig. 3b) by the following formula [1]:

$$
\mu_{2}=\mu_{1} \frac{g_{\text {plateau }}}{g_{\max }}\left(1+\frac{C_{\mathrm{Si}}}{C_{\mathrm{ox}_{1}}}+\frac{C_{i t_{1}}}{C_{\mathrm{ox}_{1}}}\right),
$$

neglecting the capacitance of the front surface states, $C_{i t_{1}}$ (see Appendix), to obtain a minimal estimation of the back channel mobility. $C_{\mathrm{Si}}$ is the capacitance of the Si film.

Transconductance derivatives, or $2^{\text {nd }}$ derivatives of transfer characteristics, with respect to $V_{G_{2}}$ are shown in Figs 3c and 3c'. The front-gate threshold voltages of the MOSFETs for front and back channels are easily determined as the positions of the peaks of these characteristics (see Fig. 4) [3].

A typical dependence of AM $p$-MOSFET front-gate channel voltages for front and back channels on conditions at the back interface is illustrated in Fig. 5a [5].
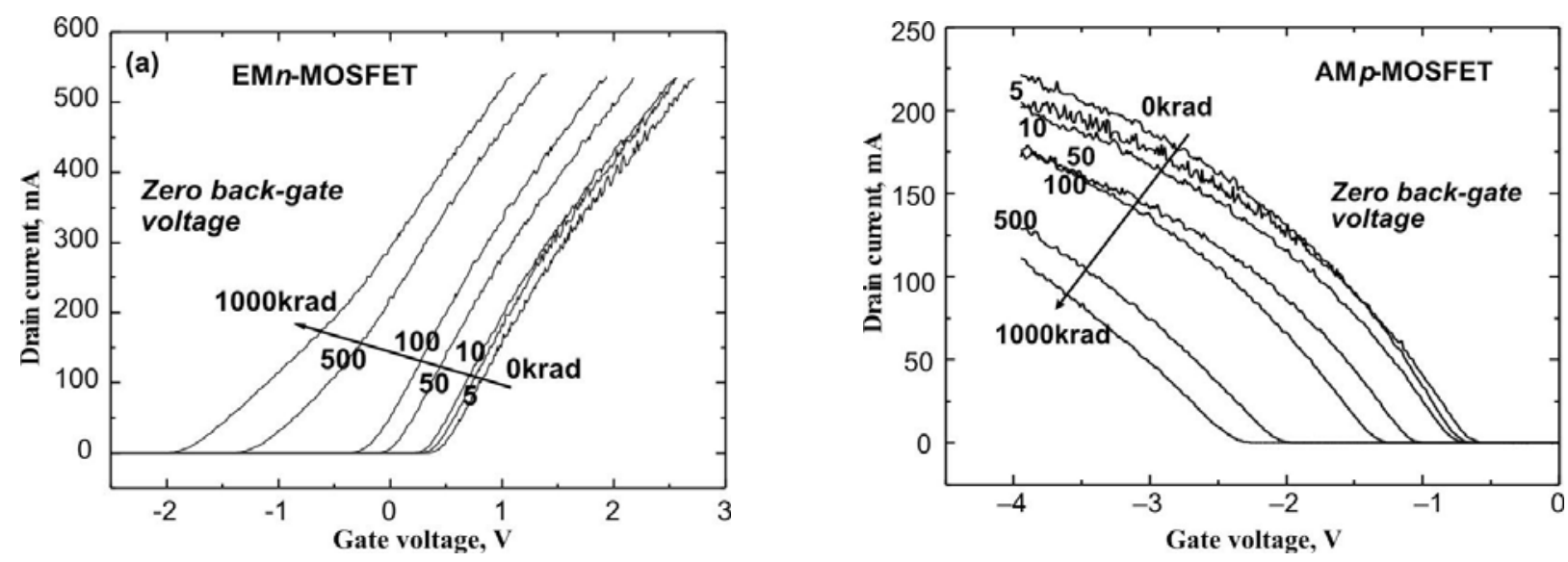

Fig. 2. Illustration of the radiation effect on MOSFETs: transfer characteristics of SOI FD EM $n$-MOSFET (a) and those of SOI AM $p$-MOSFET (b) at various radiation doses. 

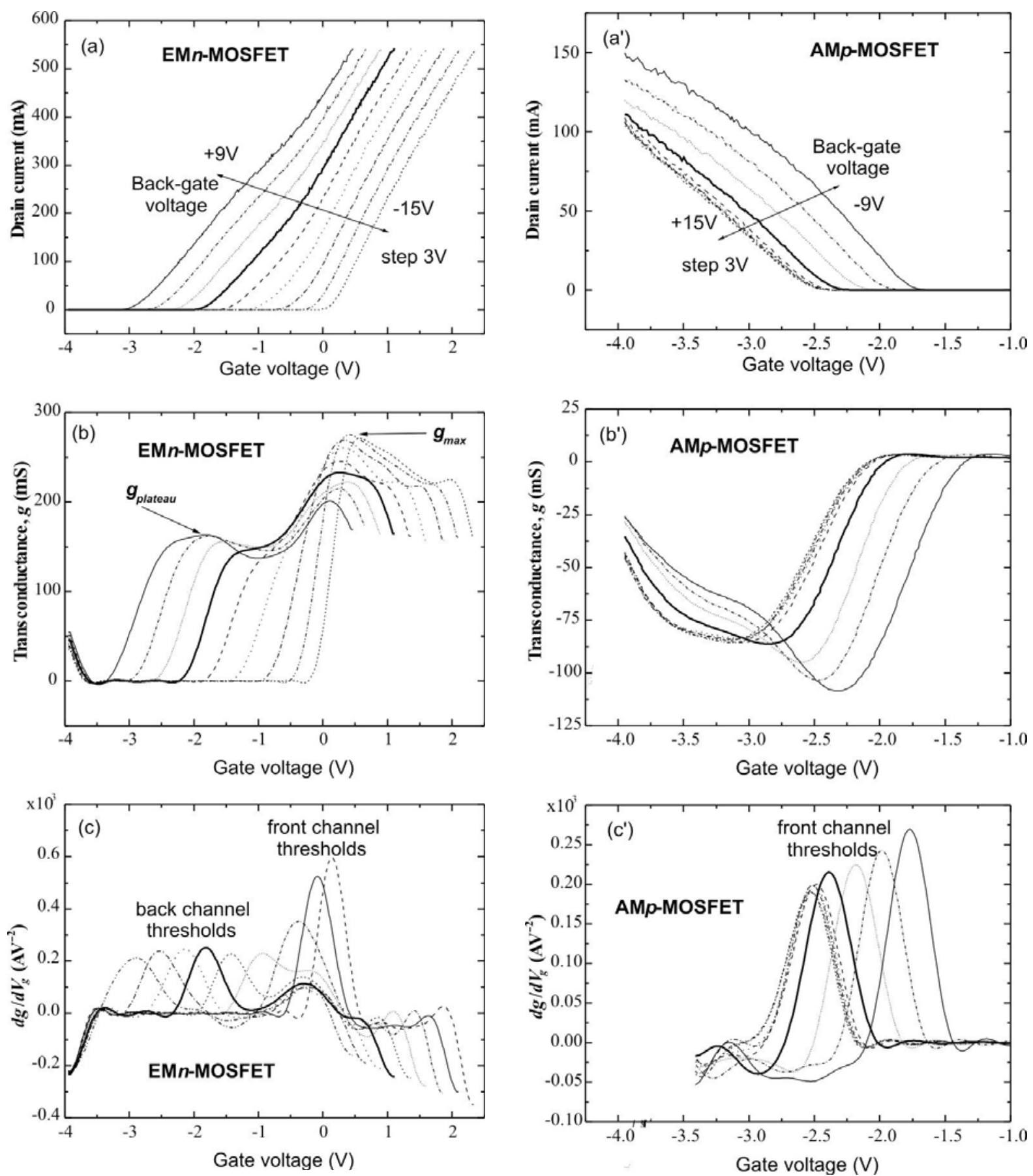

Fig. 3. $I_{D} V_{G}$ characteristics for edgeless EM $n$-MOSFET (a) and AM p-MOSFET (a'), irradiated with the total dose of $1 \cdot 10^{6} \mathrm{rad}, \mathrm{EM}(\mathrm{b})$ and AM (b') MOSFETs transconductance, and derivatives of transconductance for EM (c) and (AM) (c') MOSFETs.

From available dependences, it is possible to derive radiation-induced dynamical change of the fixed charge on the gate oxide and BOX as well as surface state capacitances for both front and back interfaces. The respective calculations can be performed most simply for the AM $p$-MOSFET, for which $I_{D} V_{G_{1}}$ characteristics are weakly affected by a back channel current, and the front channel threshold voltage can be derived easily.

Indeed, in the case of the inverted back interface $\left(\psi_{s_{2}}=2 \phi_{\mathrm{F}}\right)$ we observe the saturated $V_{\mathrm{th}_{1}}$ vs $V_{G_{2}}$ dependence for high positive $V_{G_{2}}$ values, and it is 


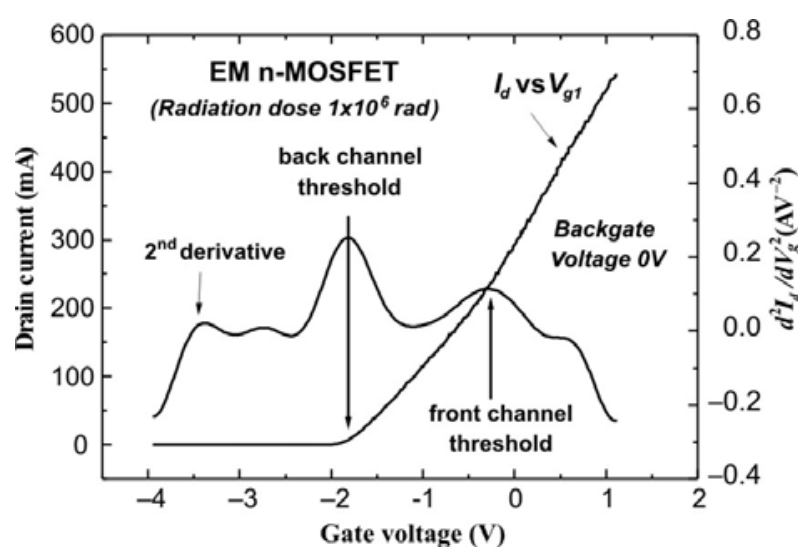

Fig. 4. The illustration of the $2^{\text {nd }}$ derivative method of threshold voltage determination.

possible to obtain the radiation-induced charge $\Delta Q_{f_{1}}$ in the gate oxide, knowing the front-channel voltage shift $\Delta V_{\mathrm{th}_{1}}$ for the interface of this type (see Fig. 5b) [5]:

$V_{\mathrm{th}_{1}}=-\frac{Q_{f_{1}}}{C_{\mathrm{ox}_{1}}}-2 \phi_{\mathrm{F}} \frac{C_{\mathrm{Si}}}{C_{\mathrm{ox}_{1}}}-\frac{Q_{\mathrm{Si}}}{2 C_{\mathrm{ox}_{1}}}$.

In the case of a depleted back interface, we have a linear decrease in the front-channel threshold voltage $V_{\text {th }}$ vs back-gate voltage $V_{G_{2}}$, which is described by the following expression [5]:

$V_{\mathrm{th}_{1}}=-\frac{Q_{f_{1}}}{C_{\mathrm{ox}_{1}}}-\frac{Q_{\mathrm{Si}}}{2 C_{\mathrm{ox}_{1}}}-\frac{C_{\mathrm{Si}} C_{\mathrm{ox}_{2}}}{C_{\mathrm{ox}_{1}}\left(C_{\mathrm{ox}_{2}}+C_{\mathrm{Si}_{1}}+C_{i t_{2}}\right)} \times$

$\times\left(V_{G_{2}}-\left[\phi_{m s_{2}}-\frac{Q_{f_{2}}}{C_{\mathrm{ox}_{2}}}-\frac{Q_{\mathrm{Si}}}{2 C_{\mathrm{ox}_{2}}}\right]\right)$.

From the slope of this linear dependence, we can obtain the capacitance of the back-interface states $C_{i t_{2}}$. Now, knowing $\Delta Q_{f_{1}}$ and $C_{i t_{2}}$ we can find the radiationinduced charge $\Delta Q_{f_{2}}$ in the BOX from the horizontal shifts $\Delta V_{G_{2}}$ of the $V_{\text {th }} v s V_{G_{2}}$ curves in the same region (see Fig. 5b). Finally, from the slope of the linear dependence of the front-gate threshold voltage for the back channel with the condition of weak accumulation at the back interface (see Fig. 5b), we can obtain the capacitance of the front-interface states $C_{i t_{1}}[5]$ :

$$
\begin{aligned}
& V_{\mathrm{th}_{2}}=-\frac{Q_{f_{1}}}{C_{\mathrm{ox}_{1}}}-\frac{Q_{\mathrm{Si}}}{2 C_{\mathrm{ox}_{1}}}-\left(1+\frac{C_{i t_{1}}+C_{\mathrm{Si}}}{C_{\mathrm{ox}_{1}}}\right) \frac{C_{\mathrm{ox}_{2}}}{C_{\mathrm{Si}}} \times \\
& \times\left(V_{G_{2}}-\left[\phi_{\mathrm{ms}_{2}}-\frac{Q_{f_{2}}}{C_{\mathrm{ox}_{2}}}-\frac{Q_{\mathrm{Si}}}{2 C_{\mathrm{ox}_{2}}}\right]\right) .
\end{aligned}
$$

\section{Results and discussion}

The threshold voltage shift in the front and back transistors at zero back-gate voltage as a function of
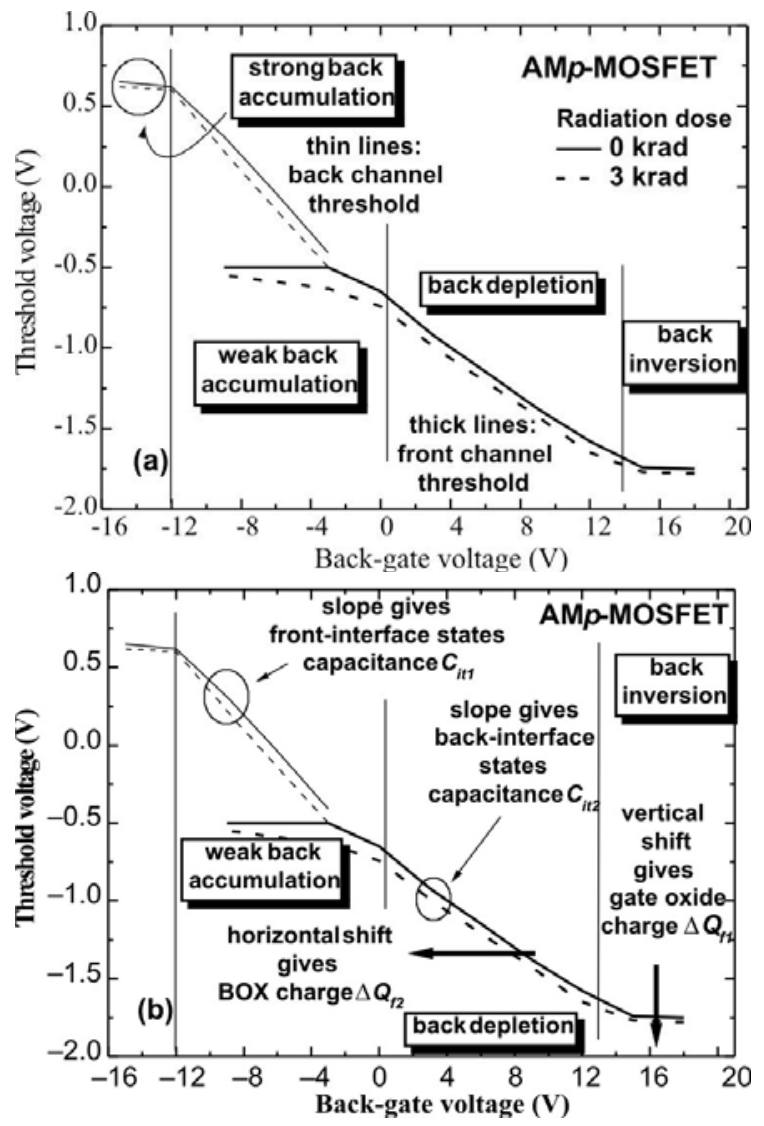

Fig. 5. Front-gate threshold voltages for front and back channels of AM p-MOSFET depending on conditions at the back interface (a) and an illustration of the radiation-induced charge parameters extraction from the dependences of the threshold voltages upon back-gate voltages (b).

irradiation dose is presented in Fig. 6 that demonstrates a stronger irradiation effect on the threshold of the front channel for the AM $p$-MOSFET than for the EM $n$ MOSFET. Such a phenomenon is possibly associated with a compensative effect of the radiation induced positive oxide charge in the gate oxide and negatively charged surface states on the threshold voltage of the EM $n$-MOSFET and the overall effect of the positive oxide charge and positively charged surface states on the threshold voltage of the AM $p$-MOSFET.

Additionally, an apparent threshold voltage shift in the MOSFETs, which is defined as the front-gate voltage from which the drain current starts to significantly increase in the linear regime [5], is also presented in Fig. 6. It should be noted that, in the case of the apparent threshold voltage shift with irradiation, the AM $p$ MOSFET appears to be more radiation-resistant than EM n-MOSFET. This is associated with inversion channel creation at the back interface of the EM nMOSFET with positive charge generation in the BOX during gamma-irradiation. Thus, for the EM $n$-MOSFET we can observe a good correlation of the shift of the apparent threshold voltage with the physical threshold voltage shift for the back channel (see Fig. 6). That is 


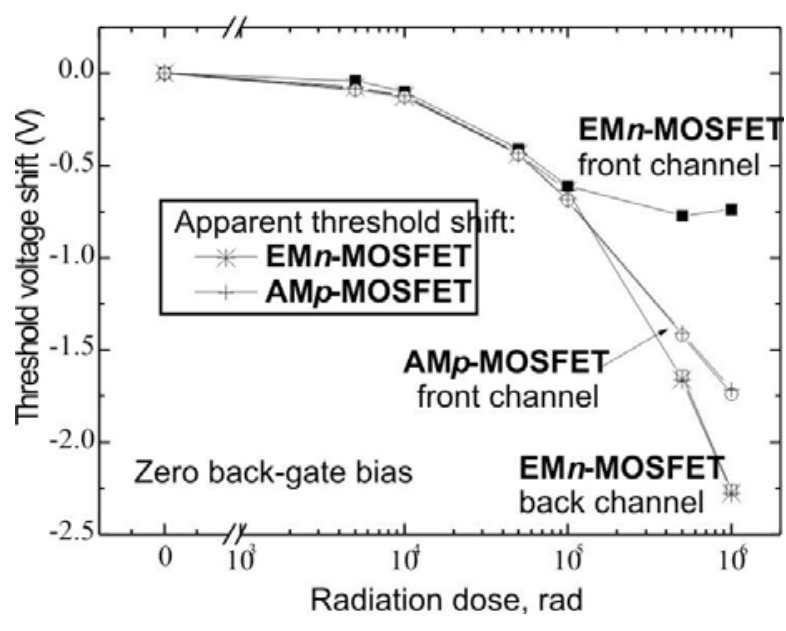

Fig. 6. Threshold voltage shifts for EM $n$ - and AM $p$ MOSFETs. The apparent threshold voltage is defined as the front-gate voltage from which the drain current starts to significantly increase in the linear regime [5].

why it is impossible to use the apparent threshold voltage to determine charges both in gate and buried oxides for EM $n$-MOSFETs.

Fig. 7 depicts the change of electron and hole mobility in the front and back channels of the MOSFETs. It should be noted that on the small dose irradiation (within the range $10^{3}$ to $10^{4} \mathrm{rad}$ ) an increase of the electron and hole mobilities is observed. This effect can be related with radiation ordering of the gate oxide/silicon film interface as well as the $\mathrm{BOX} /$ silicon film interface.

As shown in the previous section, the radiation induced charge in the gate oxide, buried oxide and interfaces can be calculated from the front channel threshold voltage $V_{\mathrm{th}_{1}}$ vs back-gate voltage $V_{G_{2}}$ characteristics as a function of the irradiation dose (see Fig. 8a). The results of the calculations for AM pMOSFET are presented in Fig. 8b. The important point is in the fact that the radiation induced positive charge in

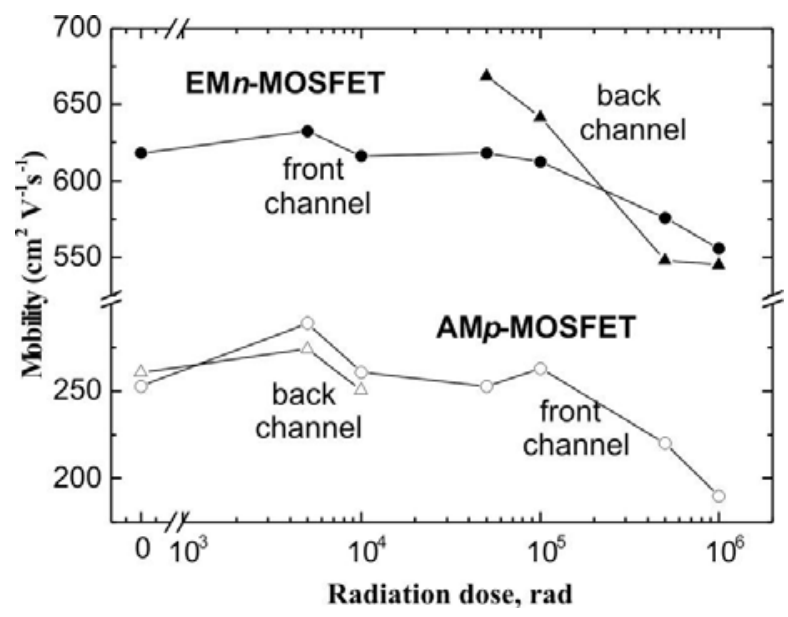

Fig. 7. The front and back channel mobilities for EM $n$ - and AM $p$-MOSFETs.
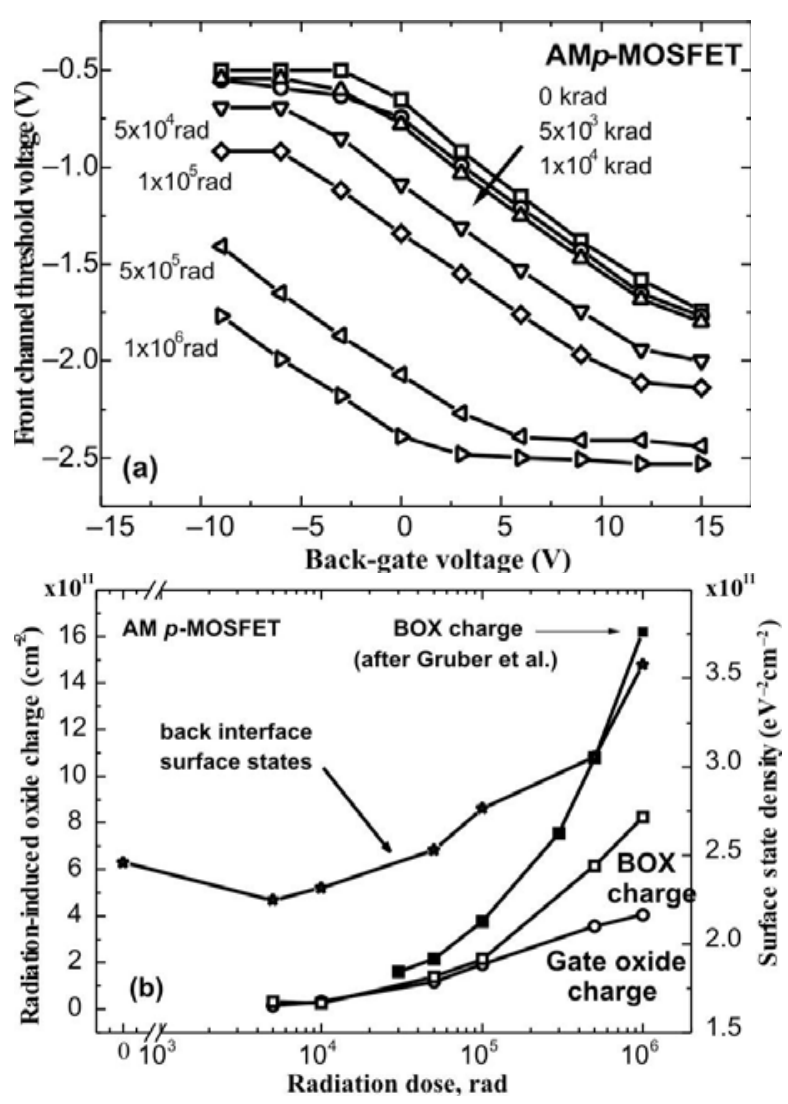

Fig. 8. The front channel threshold voltage of AM pMOSFET vs back-gate voltage for various irradiation doses (a) and radiation induced charges in the gate and buried oxides of AM p-MOSFET as well as surface state density at the BOX/Si film interface (b). Radiation induced BOX charge in MOSFET made by similar UNIBOND technology is plotted by Gruber et al. [7].

the BOX in our MOSFETs is smaller than that presented in Ref. [7] (see Fig. 8b) for similar UNIBOND technology. Fortunately, in recent years considerable efforts for developing the UNIBOND technique have improved the quality of the BOX in such SOI structures. Additionally it is noteworthy that the interface surface state density in the BOX/Si film interface reveals a minimal value in the dose range from $10^{3}$ to $10^{4} \mathrm{rad}$. This phenomenon indicates an increase of electrical quality of the back interface as well as the gate oxide/Si film interface after small dose irradiation.

\section{Conclusions}

The radiation effect on edgeless FD EM $n$-MOSFETs and AM p-MOSFETs was investigated in the present paper. The used $2^{\text {nd }}$ derivative method of threshold voltage extraction allowed to distinguish unambiguously the front-gate threshold voltages for the front and back channels and to determine the concentration of the radiation induced charges in the SOI MOSFETs by carrying out only the front-gate measurements. Following small doses of irradiation, an increase of the charge carrier 
mobility both in front and back channels and decrease of the surface state density in the BOX/Si film interface are observed, what takes place due to an increase of the quality of the structural and electrical properties of the gate oxide/film interface as well as the BOX/film interface.

\section{Appendix}

Indices 1 and 2 denote quantities corresponding to front (1) and back (2) interface.

\begin{tabular}{|l|l|}
\hline$q$ & electron charge \\
\hline$N_{a}$ & channel doping \\
\hline$L, W, t_{\mathrm{Si}}$ & $\begin{array}{l}\text { length, width and thickness of Si- } \\
\text { film }\end{array}$ \\
\hline$\phi_{m s_{1,2}}$ & $\begin{array}{l}\text { metal-semiconductor work } \\
\text { functions }\end{array}$ \\
\hline$\psi_{s_{1,2}}$ & surface potentials \\
\hline$Q_{f_{1,2}}$ & oxide fixed charges \\
\hline$Q_{c_{1,2}}$ & surface channel charges \\
\hline$Q_{\mathrm{Si}}=-q N_{a} t_{\mathrm{Si}}$ & depletion charge \\
\hline$C_{i t_{1,2}}=q D_{i t_{1,2}}$ & interface-trap capacitances \\
\hline$D_{i t_{1,2}}$ & $\begin{array}{l}\text { average concentrations of } \\
\text { interface traps }\end{array}$ \\
\hline$C_{\mathrm{ox}_{1,2}}$ & oxides capacitances \\
\hline$C_{\mathrm{Si}}$ & Si film capacitance \\
\hline$V_{G_{1,2}}$ & gate voltages \\
\hline$V_{S, D}$ & source and drain voltages \\
\hline$V_{\mathrm{th}_{1,2}}$ & threshold voltages \\
\hline$I_{D}$ & drain current \\
\hline$g$ & transconductance \\
\hline$\mu_{1,2}$ & mobilities \\
\hline
\end{tabular}

\section{Acknowledgements}

The authors thank the technical staff of the UCL Microelectronics Lab for device fabrication. This work has been supported by STCU project No 2332.

\section{References}

1. J.-P. Colinge, Silicon-on-Insulator Technology: Materials to VLSI, $3^{\text {rd }}$ edition. Kluwer Acad. Publ., Dordrecht (2004).

2. H.K. Lim and J.G. Fossum, Threshold voltage of thinfilm silicon-on-insulator (SOI) MOSFETs // IEEE Trans. Electron Devices, 39, p. 1244-1251 (1983).

3. H.-S. Wong, M.H. White, T.J. Krutsick, and R.V. Booth, Modeling of transconductance degradation and extraction of threshold voltage in thin oxide MOSFET's // Solid State Electron. 30, p. 953-968 (1987).

4. A. Terao, D. Flandre, E. Lora-Tamayo, and F. van de Wiele, Measurement of threshold voltages of thin film accumulation-mode p-MOS/SOI transistors // IEEE Electron. Dev. Lett., EDL-12, p. 682-684 (1991).

5. D. Flandre and A. Terao, Extended theoretical analysis of the steady-state linear behavior of accumulation-mode, long-channel $p$-MOSFETs on SOI substrates // Solid State Electron. 35, p. 10851092 (1992).

6. P. Paillet, J.L. Autran, O. Flament, J.L. Leray, B. Aspar, A.J. Auberton-Herve, X-radiation response of SIMOX buried oxides: influence of the fabrication process // IEEE Trans. Nucl. Sci. 43, p. 821-825 (1996).

7. O. Gruber, P. Paillet, J.L. Autran, B. Aspar, A.J. Auberton-Herve, Charge trapping in SIMOX and UNIBOND ${ }^{\circledR}$ oxides // Microelectron. Eng. 36, p. 387-390 (1997). 